\title{
Reducibility of lacunary polynomials XII
}

by

\author{
A. Schinzel (Warszawa)
}

In memory of Paul Erdős

E. Bombieri and U. Zannier [1] have recently proved an important theorem which permits improving most of the results of papers VII, VIII, X and $\mathrm{XI}$ of this series. In order to state the results I shall use the same notation as in those papers, explained below, together with a new usage of the matrix notation.

$\mathbb{N}$ and $\mathbb{N}_{0}$ are the sets of positive and non-negative integers, respectively, $\overline{\mathbb{Q}}$ is the field of algebraic numbers.

Bold face letters denote vectors written horizontally, $\boldsymbol{x}=\left[x_{1}, \ldots, x_{k}\right]$, $\boldsymbol{x}^{-1}=\left[x_{1}^{-1}, \ldots, x_{k}^{-1}\right]$ and similarly for $\boldsymbol{z} ; \boldsymbol{a} \boldsymbol{b}$ is the scalar product of $\boldsymbol{a}$ and $\boldsymbol{b}$.

The set of $k \times l$ integral matrices is denoted by $\mathfrak{M}_{k, l}(\mathbb{Z})$, and the identity matrix of order $k$ by $\boldsymbol{I}_{k}$. For a matrix $\boldsymbol{A}=\left(a_{i j}\right) \in \mathfrak{M}_{k, l}(\mathbb{Z})$ we put

$$
h(\boldsymbol{A})=\max _{i, j}\left|a_{i j}\right|, \quad \boldsymbol{x}^{\boldsymbol{A}}=\left[\prod_{i=1}^{k} x_{i}^{a_{i 1}}, \ldots, \prod_{i=1}^{k} x_{i}^{a_{i l}}\right] .
$$

For a Laurent polynomial $F \in \boldsymbol{K}\left[\boldsymbol{x}, \boldsymbol{x}^{-1}\right]$, where $\boldsymbol{K}$ is any field, if $F=$ $\prod_{i=1}^{k} x_{i}^{\alpha_{i}} F_{0}(\boldsymbol{x})$, where $F_{0} \in \boldsymbol{K}[\boldsymbol{x}]$ and $\left(F_{0}, \prod_{i=1}^{k} x_{i}\right)=1$, we put

$$
J F=F_{0} \text {. }
$$

A polynomial $F$ is reciprocal if $J F\left(\boldsymbol{x}^{-1}\right)= \pm F(\boldsymbol{x})$.

A polynomial is irreducible over $\boldsymbol{K}$ if it is not reducible over $\boldsymbol{K}$ and not a constant. For $\boldsymbol{K}=\mathbb{Q}$ we omit the words "over $\mathbb{Q}$ ". If $F=c \prod_{\sigma=1}^{s} F_{\sigma}^{e_{\sigma}}$, where $c \in \boldsymbol{K}^{*}, F_{\sigma}$ are irreducible over $\boldsymbol{K}$ and pairwise coprime, and $e_{\sigma} \geq 1$ $(1 \leq \sigma \leq s)$, we write

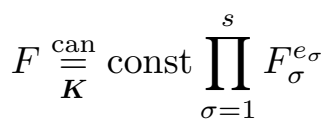

1991 Mathematics Subject Classification: Primary 11R09. 
and call this a canonical factorization of $F$ over $\boldsymbol{K}$. If $\boldsymbol{K}=\mathbb{Q}$, then $\stackrel{\overline{\bar{K}}}{\overline{\mathrm{can}}}$ is replaced by $\stackrel{\text { can }}{=}$. If

$$
J F \stackrel{\text { can }}{\bar{K}} \text { const } \prod_{\sigma=1}^{s} F_{\sigma}^{e_{\sigma}}
$$

we put

and if $\boldsymbol{K}=\mathbb{Q}$

$$
K F=\mathrm{const} \prod^{*} F_{\sigma}^{e_{\sigma}},
$$

$$
L F=\text { const } \prod^{* *} F_{\sigma}^{e_{\sigma}},
$$

where $\prod^{*}$ is taken over all $F_{\sigma}$ that do not divide $J\left(\boldsymbol{x}^{t} \boldsymbol{\alpha}-1\right)$ for any $\boldsymbol{\alpha} \in$ $\mathbb{Z}^{k} \backslash\{\mathbf{0}\}$ and $\prod^{* *}$ is taken over all $F_{\sigma}$ that are not reciprocal. The leading coefficients (i.e. the coefficients of the first term in the antilexicographic order) of $K F$ and $L F$ are equal to that of $F$. Note that $K F$ depends only on $F$ and the prime field of $\boldsymbol{K}$, which in this paper is always $\mathbb{Q}$. then

If $T$ is any transformation of $\boldsymbol{K}\left[\boldsymbol{x}, \boldsymbol{x}^{-1}\right]$ into itself and $F \in \boldsymbol{K}\left[\boldsymbol{x}, \boldsymbol{x}^{-1}\right]$

and if $\boldsymbol{K}=\mathbb{Q}$

$$
K F(T \boldsymbol{x})=K(F(T \boldsymbol{x}))
$$

$$
L F(T \boldsymbol{x})=L(F(T \boldsymbol{x})) .
$$

The Bombieri-Zannier theorem can be stated as follows.

Theorem BZ. Let $P, Q \in \overline{\mathbb{Q}}[\boldsymbol{x}]$ and $\boldsymbol{n} \in \mathbb{Z}^{k}$. If $(P, Q)=1$, but $\left(K P\left(x^{n}\right), K Q\left(x^{n}\right)\right) \neq 1$, then there exists a $\gamma \in \mathbb{Z}^{k}$ such that

$$
\boldsymbol{\gamma} \boldsymbol{n}=0 \quad \text { and } \quad 0<h(\gamma) \leq c_{1}(P, Q),
$$

where $c_{1}(P, Q)$ depends only on $P$ and $Q$.

In the sequel $c_{i}(\ldots)$ denote effectively computable positive numbers depending only on parameters displayed in parentheses. Theorem BZ extends Theorem 1 of [7] from $k \leq 3$ to arbitrary $k$ in the crucial case $[\boldsymbol{K}: \mathbb{Q}]<\infty$ and immediately implies that in Theorem 2 of [7],

$$
c_{2}(P, Q) N^{k-\min \{k, 6\} /(2 k-2)} \frac{(\log N)^{10}}{(\log \log N)^{9}}
$$

can be replaced by

$$
c_{2}(P, Q) N^{k-1} .
$$

Theorems 3 and 5 of [7] can now be extended in the following manner.

Theorem 1. Let $F \in \mathbb{Z}[\boldsymbol{x}] \backslash\{0\}, k_{0}$ be the number of variables with respect to which $F$ is of positive degree, and $\|F\|$ be the sum of squares of the coefficients of $F$. Assume $K F=L F$. For every vector $\boldsymbol{n} \in \mathbb{Z}^{k}$ such that 
$F\left(x^{n}\right) \neq 0$ there exist a matrix $\boldsymbol{M}=\left(\mu_{i j}\right) \in \mathfrak{M}_{k, k}(\mathbb{Z})$ and a vector $\boldsymbol{v} \in \mathbb{Z}^{k}$ such that

$$
\begin{gathered}
0 \leq \mu_{i j}<\mu_{j j} \leq \exp \left(9 k_{0}\right) \cdot 2^{\|F\|-5} \quad(i \neq j), \quad \mu_{i j}=0 \quad(i<j), \\
\boldsymbol{n}=\boldsymbol{v} \boldsymbol{M},
\end{gathered}
$$

and either

$$
K F\left(\boldsymbol{z}^{M}\right) \stackrel{\text { can }}{=} \text { const } \prod_{\sigma=1}^{s} F_{\sigma}(\boldsymbol{z})^{e_{\sigma}}
$$

implies

$$
K F\left(x^{n}\right) \stackrel{\text { can }}{=} \text { const } \prod_{\sigma=1}^{s} F_{\sigma}\left(x^{v}\right)^{e_{\sigma}},
$$

or there exists a $\gamma \in \mathbb{Z}^{k}$ such that

$$
\boldsymbol{\gamma} \boldsymbol{n}=0 \quad \text { and } \quad 0<h(\gamma) \leq c_{3}(F, \boldsymbol{M}) .
$$

Theorem 4 of [7] is extended as follows.

Theorem 2. Let $F \in \mathbb{Q}[\boldsymbol{x}] \backslash\{0\}$ and $\boldsymbol{n} \in \mathbb{Z}^{k} \backslash\{\mathbf{0}\}$. If $J F\left(x^{\boldsymbol{n}}\right)$ is not reciprocal, then $K F\left(x^{n}\right)$ is reducible if and only if there exists a matrix $\boldsymbol{N} \in \mathfrak{M}_{r, k}(\mathbb{Z})$ of rank $r$ and a vector $\boldsymbol{v} \in \mathbb{Z}^{r}$ such that

$$
\begin{gathered}
h(\boldsymbol{N}) \leq c_{4}(F), \\
\boldsymbol{n}=\boldsymbol{v} \boldsymbol{N}, \\
K F\left(\boldsymbol{y}^{\boldsymbol{N}}\right)=F_{1} F_{2}, \quad \boldsymbol{y}=\left[y_{1}, \ldots, y_{r}\right], \quad F_{i} \in \mathbb{Q}[\boldsymbol{y}] \quad(i=1,2), \\
K F_{i}\left(x^{\boldsymbol{v}}\right) \notin \mathbb{Q} \quad(i=1,2) .
\end{gathered}
$$

Further we have

Theorem 3. Let $F \in \overline{\mathbb{Q}}[\boldsymbol{x}] \backslash\{0\}, \boldsymbol{n} \in \mathbb{Z}^{k} \backslash\{\mathbf{0}\}, \boldsymbol{K}$ be the field generated over $\mathbb{Q}$ by the ratios of the coefficients of $F\left(x^{n}\right)$ and $\widehat{\boldsymbol{K}}$ be its normal closure. Assume that $F \in \boldsymbol{K}[\boldsymbol{x}], F\left(x^{\boldsymbol{n}}\right) \neq 0$ and for all embeddings $\tau$ of $\boldsymbol{K}$ into $\widehat{\boldsymbol{K}}$,

$$
\frac{J F\left(x^{-n}\right)}{J F^{\tau}\left(x^{n}\right)} \notin \widehat{\boldsymbol{K}} .
$$

If $K F\left(x^{n}\right)$ is reducible over $\boldsymbol{K}$ there exist a matrix $\boldsymbol{N} \in \mathfrak{M}_{r, k}(\mathbb{Z})$ of rank $r$ and a vector $\boldsymbol{v} \in \mathbb{Z}^{r}$ such that

$$
\begin{gathered}
h(\boldsymbol{N}) \leq c_{5}(F), \\
\boldsymbol{n}=\boldsymbol{v} \boldsymbol{N}
\end{gathered}
$$

and $J F\left(\boldsymbol{y}^{\boldsymbol{N}}\right)$ is reducible over $\widehat{\boldsymbol{K}}$, where $\boldsymbol{y}=\left[y_{1}, \ldots, y_{r}\right]$. 
This theorem implies

Corollary 1. Let $\boldsymbol{a}=\left[a_{0}, \ldots, a_{k}\right] \in \overline{\mathbb{Q}}^{* k+1}, \boldsymbol{n}=\left[n_{1}, \ldots, n_{k}\right] \in \mathbb{N}^{k}$, $0<n_{1}<\ldots<n_{k}$ and let $\boldsymbol{K}=\mathbb{Q}\left(a_{1} / a_{0}, \ldots, a_{k} / a_{0}\right)$. If $a_{0} \in \boldsymbol{K}$ and $K\left(a_{0}+\sum_{j=1}^{k} a_{j} x^{n_{j}}\right)$ is reducible over $\boldsymbol{K}$, then there exist a matrix $\boldsymbol{N}_{0} \in$ $\mathfrak{M}_{[(k+1) / 2], k}(\mathbb{Z})$ and a vector $\boldsymbol{v}_{0} \in \mathbb{Z}^{[(k+1) / 2]}$ such that

$$
h\left(\boldsymbol{N}_{0}\right) \leq c_{6}(\boldsymbol{a})
$$

and

$$
n=\boldsymbol{v}_{0} \boldsymbol{N}_{0}
$$

COROLlaRY 2. Under the assumptions of Corollary 1 the number of vectors $\boldsymbol{n}$ such that $n_{k} \leq N$ and $K\left(a_{0}+\sum_{j=1}^{k} a_{j} x^{n_{j}}\right)$ is reducible over $\boldsymbol{K}$ is less than $c_{7}(\boldsymbol{a}) N^{[(k+1) / 2]}$.

Corollary 3. Let $\boldsymbol{a}=\left[a_{0}, \ldots, a_{k}\right] \in \mathbb{C}^{* k+1}$ be such that $a_{0} \in \boldsymbol{K}=$ $\mathbb{Q}\left(a_{1} / a_{0}, \ldots, a_{k} / a_{0}\right)$. The number of integer vectors $\boldsymbol{n}=\left[n_{1}, \ldots, n_{k}\right]$ such that $0<n_{1}<\ldots<n_{k} \leq N$ and $K\left(a_{0}+\sum_{j=1}^{k} a_{j} x^{n_{j}}\right)$ is reducible over $\boldsymbol{K}$ is less than $c_{8}(\boldsymbol{a}) N^{k-1}$.

Corollary 1 improves in the case $\boldsymbol{K}=\mathbb{Q}$ and extends Theorem 2 of [3], while Corollary 2 drastically improves Theorem 1 of [5]. The exponent $[(k+1) / 2]$ cannot be further improved, as will be shown by an example, the gist of which is in [3]. Corollary 3 improves Theorem 2 of [6] and the Theorem of [8].

Further we have

Theorem 4. Let $F \in \mathbb{Q}[\boldsymbol{x}] \backslash\{0\}$. There exist two finite subsets $R$ and $S$ of $\bigcup_{r=1}^{k} \mathfrak{M}_{r, k}(\mathbb{Z})$ with the following property. If $\boldsymbol{n} \in \mathbb{Z}^{k} \backslash\{\mathbf{0}\}$ and $J F\left(x^{\boldsymbol{n}}\right)$ is not reciprocal, then $K F\left(x^{\boldsymbol{n}}\right)$ is reducible if and only if the equation $\boldsymbol{n}=\boldsymbol{v} \boldsymbol{N}$ is soluble in $\boldsymbol{v} \in \mathbb{Z}^{r}$ and $\boldsymbol{N} \in R \cap \mathfrak{M}_{r, k}(\mathbb{Z})$ but unsoluble in $\boldsymbol{v} \in \mathbb{Z}^{s}$ and $\boldsymbol{N} \in S \cap \mathfrak{M}_{s, k}(\mathbb{Z})$ for each $s<r$.

The reducibility condition given in Theorem 4 is more readily verifiable than that of Theorem 2, because of the relation (9) occurring in the latter. It is conjectured that a similar reducibility condition holds without the assumption that $J F\left(x^{n}\right)$ is not reciprocal and over any finite extension of $\mathbb{Q}$.

The proofs of Theorems 1-4 are based on several lemmas.

Lemma 1. For every polynomial $P \in \mathbb{Q}[\boldsymbol{x}] \backslash\{0\}$,

$$
L K P=L P .
$$

Pro of. See [2], Lemma 11.

Lemma 2. For every polynomial $F \in \mathbb{Z}[\boldsymbol{x}]$ and every vector $\boldsymbol{n} \in \mathbb{Z}^{k}$ such that $F\left(x^{n}\right) \neq 0$ there exist a matrix $\boldsymbol{M}=\left(\mu_{i j}\right) \in \mathfrak{M}_{k, k}(\mathbb{Z})$ and a vector 
$\boldsymbol{v} \in \mathbb{Z}^{k}$ such that

(15) $\quad 0 \leq \mu_{i j}<\mu_{j j} \leq \exp (9 k) \cdot 2^{\|F\|-5} \quad(i \neq j), \quad \mu_{i j}=0 \quad(i<j)$,

$$
\boldsymbol{n}=\boldsymbol{v} M,
$$

and either

$$
L F\left(\boldsymbol{z}^{M}\right) \stackrel{\text { can }}{=} \text { const } \prod_{\sigma=1}^{s} F_{\sigma}^{e_{\sigma}}
$$

implies

$$
L F\left(x^{n}\right) \stackrel{\text { can }}{=} \text { const } \prod_{\sigma=1}^{s} F_{\sigma}\left(x^{v}\right)^{e_{\sigma}},
$$

or there exists a vector $\gamma \in \mathbb{Z}^{k}$ such that

$$
\boldsymbol{\gamma} \boldsymbol{n}=0 \quad \text { and } \quad 0<h(\boldsymbol{\gamma}) \leq c_{9}(k, F) .
$$

Proof. See [2], Lemma 12, where $c_{9}(k, F)$ is given explicitly.

LEMMA 3. If $F \in \mathbb{Q}[\boldsymbol{x}]$ is irreducible and non-reciprocal and a matrix $\boldsymbol{M} \in \mathfrak{M}_{k, k}(\mathbb{Z})$ is non-singular, then

$$
L F\left(\boldsymbol{z}^{M}\right)=J F\left(\boldsymbol{z}^{M}\right) .
$$

Proof. See [7], Lemma 17. then

$$
K F\left(\boldsymbol{z}^{\boldsymbol{M}}\right)=L F\left(\boldsymbol{z}^{\boldsymbol{M}}\right) .
$$

Pr o of. By Lemma 1 we have, for every polynomial $P \in \mathbb{Q}[\boldsymbol{x}] \backslash\{0\}$,

$$
L P|K P| J P \text {. }
$$

Assume first that $F$ is irreducible. If $F=c x_{i}, c \in \mathbb{Q}$, then $J F\left(\boldsymbol{z}^{M}\right)=c$, hence $K F\left(\boldsymbol{z}^{\boldsymbol{M}}\right)=L F\left(\boldsymbol{z}^{\boldsymbol{M}}\right)=c$. If $F \mid J\left(\boldsymbol{x}^{t} \boldsymbol{\alpha}-1\right)$ for an $\boldsymbol{\alpha} \in \mathbb{Z}^{k} \backslash\{\mathbf{0}\}$, then $F\left(\boldsymbol{z}^{\boldsymbol{M}}\right) \mid J\left(\boldsymbol{z}^{\boldsymbol{M}^{t} \boldsymbol{\alpha}}-1\right)$, hence $K F\left(\boldsymbol{z}^{\boldsymbol{M}}\right) \in \mathbb{Q}$ and (18) implies (17). If $F \neq c x_{i}$ for all $c \in \mathbb{Q}$ and all $i \leq k$, and $F \nmid J\left(\boldsymbol{x}^{t} \boldsymbol{\alpha}-1\right)$ for all $\boldsymbol{\alpha} \in \mathbb{Z}^{k} \backslash\{\boldsymbol{0}\}$, then $K F=F$, hence $K F=L F$ implies that $F$ is not reciprocal. By Lemma 3 we have $L F\left(\boldsymbol{z}^{\boldsymbol{M}}\right)=J F\left(\boldsymbol{z}^{\boldsymbol{M}}\right)$ and (18) implies (17).

Assume now that

$$
F \stackrel{\text { can }}{=} c \prod_{\sigma=1}^{s} F_{\sigma}^{e_{\sigma}}, \quad c \in \mathbb{Q}^{*} .
$$

Then

$$
K F=c \prod_{\sigma=1}^{s} K F_{\sigma}^{e_{\sigma}}, \quad L F=c \prod_{\sigma=1}^{s} L F_{\sigma}^{e_{\sigma}}
$$


which together with $K F=L F$ and (18) implies

$$
K F_{\sigma}=L F_{\sigma} \quad(1 \leq \sigma \leq s) .
$$

By the part of the lemma already proved, $K F_{\sigma}\left(\boldsymbol{z}^{M}\right)=L F_{\sigma}\left(\boldsymbol{z}^{M}\right)$, hence

$$
K F\left(\boldsymbol{z}^{\boldsymbol{M}}\right)=c \prod_{\sigma=1}^{s} K F_{\sigma}\left(\boldsymbol{z}^{\boldsymbol{M}}\right)^{e_{\sigma}}=c \prod_{\sigma=1}^{s} L F_{\sigma}\left(\boldsymbol{z}^{\boldsymbol{M}}\right)^{e_{\sigma}}=L F\left(\boldsymbol{z}^{\boldsymbol{M}}\right) .
$$

Lemma 5. Let $\Phi \in \mathbb{Q}[x]$ be irreducible, $\gamma=\left(\gamma_{1}, \ldots, \gamma_{k}\right) \in \mathbb{Z}^{k}$ and $\left(\gamma_{1}, \ldots, \gamma_{k}\right)=1$. Then $J \Phi\left(\boldsymbol{x}^{t} \gamma\right)$ is irreducible.

Proof. See [4], Lemma 11.

Lemma 6. If $F \in \mathbb{Q}[\boldsymbol{x}]$ and $K F \in \mathbb{Q}$, then for every vector $\boldsymbol{v} \in \mathbb{Z}^{k}$ we have $K F\left(x^{v}\right) \in \mathbb{Q}$.

Proof. It is enough to prove the lemma for $F$ irreducible and different from $c x_{i}(1 \leq i \leq k), c \in \mathbb{Q}^{*}$. The condition $K F \in \mathbb{Q}$ gives

$$
F \mid J\left(\boldsymbol{x}^{t} \boldsymbol{\alpha}-1\right), \quad \text { where } \boldsymbol{\alpha} \in \mathbb{Z}^{k} \backslash\{\mathbf{0}\} .
$$

If $\boldsymbol{\alpha} \boldsymbol{v} \neq 0$ the conclusion follows at once, but the case $\boldsymbol{\alpha} \boldsymbol{v}=0$ remains to be considered.

Let $\boldsymbol{\alpha}=a \boldsymbol{\gamma}$, where $a \in \mathbb{N}, \boldsymbol{\gamma} \in \mathbb{Z}^{k}$ and the coordinates of $\boldsymbol{\gamma}$ are relatively prime. We have

$$
J\left(\boldsymbol{x}^{t} \boldsymbol{\alpha}-1\right)=\prod_{d \mid a} J \phi_{d}\left(\boldsymbol{x}^{t} \gamma\right),
$$

where $\phi_{d}$ is the cyclotomic polynomial of order $d$. By Lemma $5, J \phi_{d}\left(\boldsymbol{x}^{t} \boldsymbol{\gamma}\right)$ is irreducible. Hence $F=c J \phi_{d}\left(\boldsymbol{x}^{t} \gamma\right)$ for a $c \in \mathbb{Q}^{*}$ and a divisor $d$ of $a$. The equality $\boldsymbol{\alpha} \boldsymbol{v}=0$ gives $\boldsymbol{v}^{t} \boldsymbol{\gamma}=(0)$, hence $J F\left(x^{\boldsymbol{v}}\right)=c \phi_{d}(1) \in \mathbb{Q}$.

Proof of Theorem 1. Let $c_{1}$ have the meaning of Theorem BZ and $c_{9}$ the meaning of Lemma 2. We may assume without loss of generality that $F \in \mathbb{Q}\left[x_{1}, \ldots, x_{k_{0}}\right]$ and apply Lemma 2 with $k$ replaced by $k_{0}, \boldsymbol{n}$ replaced by $\boldsymbol{n}_{0}=\left[n_{1}, \ldots, n_{k_{0}}\right]$, and $\boldsymbol{z}$ replaced by $\boldsymbol{z}_{0}=\left[z_{1}, \ldots, z_{k_{0}}\right]$. Let $\boldsymbol{M}_{0}$ and $\boldsymbol{v}_{0}$ be the matrix and the vector the existence of which is asserted in Lemma 2. We put

$$
\begin{array}{cl}
\left(\mu_{i j}\right)_{i, j \leq k_{0}}=\boldsymbol{M}_{0}, \quad \mu_{i i}=1 \text { if } i>k_{0}, & \mu_{i j}=0 \text { if } i>k_{0} \text { or } j>k_{0} \text { and } i \neq j ; \\
{\left[v_{1}, \ldots, v_{k_{0}}\right]=\boldsymbol{v}_{0},} & v_{i}=n_{i} \text { if } i>k_{0} .
\end{array}
$$

This together with (15) and (16) gives (1) and (2). Moreover, by Lemma 2, either

$$
L F\left(\boldsymbol{z}^{\boldsymbol{M}}\right)=L F\left(\boldsymbol{z}_{0}^{M_{0}}\right) \stackrel{\text { can }}{=} \text { const } \prod_{\sigma=1}^{s_{0}} F_{\sigma}^{0}\left(\boldsymbol{z}_{0}\right)^{e_{\sigma}^{0}}
$$


implies

$$
L F\left(x^{n}\right)=L F\left(x^{n_{0}}\right) \stackrel{\text { can }}{=} \text { const } \prod_{\sigma=1}^{s_{0}} F_{\sigma}^{0}\left(x^{v_{0}}\right)^{e_{\sigma}^{0}},
$$

or there exists a $\gamma_{0} \in \mathbb{Z}^{k_{0}}$ such that

$$
\gamma_{0} \boldsymbol{n}_{0}=0 \quad \text { and } \quad 0<h\left(\gamma_{0}\right) \leq c_{9}\left(k_{0}, F\right) .
$$

By Lemma 4 the left-hand sides of (3) and (19) coincide. Since the canonical factorization is essentially unique we have $s=s_{0}$ and we may assume that $F_{\sigma}=F_{\sigma}^{0}, e_{\sigma}=e_{\sigma}^{0}(1 \leq \sigma \leq s)$. Therefore $\left(J F_{\sigma}\left(\boldsymbol{z}^{-1}\right), F_{\sigma}(\boldsymbol{z})\right)=1$ for all $\sigma \leq s$ and the number

$$
c_{3}(F, \boldsymbol{M})=\max \left\{c_{9}\left(k_{0}, F\right), \max _{1 \leq \sigma \leq s} c_{1}\left(J F_{\sigma}\left(\boldsymbol{z}^{-1}\right), F_{\sigma}(\boldsymbol{z})\right)\right\}
$$

is well defined. We now show that it has the property claimed in the theorem.

By (3) we have

$$
F\left(\boldsymbol{z}^{\boldsymbol{M}}\right)=F_{0}(\boldsymbol{z}) \prod_{\sigma=1}^{s} F_{\sigma}(\boldsymbol{z})^{e_{\sigma}}
$$

where $K F_{0} \in \mathbb{Q}$. Hence on substitution $\boldsymbol{z}=x^{\boldsymbol{v}}$ we obtain, by (2),

$$
F\left(x^{n}\right)=F_{0}\left(x^{v}\right) \prod_{\sigma=1}^{s} F_{\sigma}\left(x^{v}\right)^{e_{\sigma}},
$$

and, on applying $K$ to both sides, by Lemma 6 we infer that

$$
K F\left(x^{n}\right)=\mathrm{const} \prod_{\sigma=1}^{s} K F_{\sigma}\left(x^{v}\right)^{e_{\sigma}} .
$$

If $K F_{\sigma}\left(x^{v}\right)=L F_{\sigma}\left(x^{v}\right)$ for all $\sigma \leq s$, then since $F_{\sigma}\left(x^{v}\right)=F_{\sigma}^{0}\left(x^{v_{0}}\right)$, (20) implies (4), while (21) and (22) imply (5) with $\gamma=\left[\gamma_{0}, 0, \ldots, 0\right]$. If $K F_{\sigma}\left(x^{v}\right) \neq L F_{\sigma}\left(x^{v}\right)$ for at least one $\sigma \leq s$, then $K F_{\sigma}\left(x^{v}\right)$ has an irreducible reciprocal factor. Hence

$$
\left(K F_{\sigma}\left(x^{-\boldsymbol{v}}\right), K F_{\sigma}\left(x^{v}\right)\right) \neq 1
$$

and by Theorem BZ there is a $\gamma \in \mathbb{Z}^{k}$ such that $\gamma \boldsymbol{n}=0$ and $0<h(\gamma) \leq$ $c_{1}\left(J F_{\sigma}\left(\boldsymbol{z}^{-1}\right), F_{\sigma}(\boldsymbol{z})\right)$, which gives (5) by virtue of $(22)$.

Lemma 7. Let $F \in \mathbb{Q}[\boldsymbol{x}]$ with $K F \notin \mathbb{Q}$. If $\boldsymbol{n} \in \mathbb{Z}^{k}$ and $K F\left(x^{\boldsymbol{n}}\right) \in \mathbb{Q}$, then there exists a vector $\gamma \in \mathbb{Z}^{k}$ such that

$$
\boldsymbol{\gamma} \boldsymbol{n}=0 \quad \text { and } \quad 0<h(\boldsymbol{\gamma}) \leq c_{10}(F) .
$$

Proof. See [7], Lemma 18.

Lemma 8. Let $G \in \overline{\mathbb{Q}}\left[\boldsymbol{x} \backslash \backslash\{0\}, \boldsymbol{n} \in \mathbb{Z}^{k} \backslash\{\mathbf{0}\}, \boldsymbol{K}\right.$ be the field generated over $\mathbb{Q}$ by the ratios of the coefficients of $G\left(x^{n}\right)$ and $\widehat{\boldsymbol{K}}$ be its normal closure. 
Assume that $G \in \boldsymbol{K}[\boldsymbol{x}], G\left(x^{n}\right) \neq 0$ and

$$
J G\left(x^{-n}\right) / J G^{\tau}\left(x^{n}\right) \notin \widehat{\boldsymbol{K}} \quad \text { for all embeddings } \tau \text { of } \boldsymbol{K} \text { into } \widehat{\boldsymbol{K}} .
$$

There exist a matrix $\boldsymbol{M} \in \mathfrak{M}_{k, k}(\mathbb{Z})$ and a vector $\boldsymbol{v} \in \mathbb{Z}^{k}$ such that

$$
\begin{gathered}
\operatorname{det} \boldsymbol{M \neq 0 ,} \quad h(\boldsymbol{M}) \leq c_{11}(G), \\
\boldsymbol{n}=\boldsymbol{v} \boldsymbol{M},
\end{gathered}
$$

and either

$$
K G\left(x^{n}\right) \text { is irreducible over } \boldsymbol{K},
$$

or there exists a vector $\gamma \in \mathbb{Z}^{k}$ such that

$$
\boldsymbol{\gamma} \boldsymbol{n}=0 \quad \text { and } \quad 0<h(\gamma) \leq c_{12}(G)
$$

or

$$
J G\left(\boldsymbol{z}^{M}\right)=G_{1} G_{2}, \quad G_{i} \in \widehat{\boldsymbol{K}}[\boldsymbol{z}] \backslash \widehat{\boldsymbol{K}}
$$

and if $\boldsymbol{K}=\mathbb{Q}$

$$
K G_{i}\left(x^{v}\right) \notin \mathbb{Q} \quad(i=1,2) .
$$

Pro of. Let $T$ be the set of all embeddings of $\boldsymbol{K}$ into $\widehat{\boldsymbol{K}}$. The assumption (25) implies

$$
\frac{J G\left(\boldsymbol{x}^{-1}\right)}{J G^{\tau}(\boldsymbol{x})} \notin \widehat{\boldsymbol{K}} \quad \text { for all } \tau \in T
$$

hence, in particular, $J G \notin \widehat{\boldsymbol{K}}$. If $J G$ is reducible over $\widehat{\boldsymbol{K}}$ or $\boldsymbol{K}=\mathbb{Q}$ and $K G$ is reducible we have (26), (27) and (30) with $\boldsymbol{M}=\boldsymbol{I}_{k}, \boldsymbol{v}=\boldsymbol{n}$ (provided $\left.c_{11}(G) \geq 1\right)$ and for $\boldsymbol{K}=\mathbb{Q}$ we may additionally assume that

$$
K G_{i} \notin \mathbb{Q} \quad(i=1,2) .
$$

In this last case we have either (31) or, denoting by $l_{i}$ the leading coefficient of $G$,

$$
K l_{i}^{-1} G_{i}\left(x^{n}\right) \in \mathbb{Q} \quad \text { for an } i \leq 2 .
$$

However, $l_{i}^{-1} G_{i}$ belongs to a finite set $S$ of monic non-constant divisors $D$ of $J G$ in $\mathbb{Q}[\boldsymbol{z}]$ satisfying $K D \notin \mathbb{Q}$ by virtue of (33). Hence, by Lemma 7 , (29) holds provided

$$
c_{12}(G) \geq \max _{D \in S} c_{10}(D) .
$$

It remains to consider the case where $J G$ is irreducible over $\widehat{\boldsymbol{K}}$, or $\boldsymbol{K}=\mathbb{Q}$ and $K G$ is irreducible.

If $J G$ is irreducible over $\widehat{\boldsymbol{K}}$, let $l$ be the leading coefficient of $J G\left(x^{n}\right)$. Since $J G\left(x^{n}\right)$ has the same coefficients as $G\left(x^{n}\right)$, by the definition of $\boldsymbol{K}$, $\tau_{1} \neq \tau_{2}$ implies that for all $\tau_{1}, \tau_{2} \in T$,

$$
\left(l^{-1} J G\left(x^{n}\right)\right)^{\tau_{1}} \neq\left(l^{-1} J G\left(x^{n}\right)\right)^{\tau_{2}}
$$


and since both sides are monic,

$$
\frac{\left(l^{-1} J G\left(x^{n}\right)\right)^{\tau_{2}}}{\left(l^{-1} J G\left(x^{n}\right)\right)^{\tau_{1}}} \notin \widehat{\boldsymbol{K}} .
$$

It follows that $J G^{\tau_{2}} / J G^{\tau_{1}} \notin \widehat{\boldsymbol{K}}$, and since $J G^{\tau_{1}}, J G^{\tau_{2}}$ are both irreducible over $\widehat{\boldsymbol{K}},\left(J G^{\tau_{1}}, J G^{\tau_{2}}\right)=1$. If $F$ is the polynomial over $\mathbb{Z}$ with the least positive leading coefficient divisible by $J G$ and irreducible over $\mathbb{Q}$ we find that

$$
J N_{K / \mathbb{Q}} G=\prod_{\tau \in T} J G^{\tau} \mid F
$$

and, since $J N_{\boldsymbol{K} / \mathbb{Q}} G \in \mathbb{Q}[\boldsymbol{x}] \backslash \mathbb{Q}$, we infer that

$$
J N_{K / \mathbb{Q}} G / F \in \mathbb{Q}^{*} .
$$

Moreover, by (32),

$$
\left(J F\left(\boldsymbol{x}^{-1}\right), F\right)=1,
$$

which implies $L F=F$ and, by (18), $K F=L F$.

If $\boldsymbol{K}=\mathbb{Q}$ and $K G$ is irreducible we define $F$ as the polynomial over $\mathbb{Z}$ which is a scalar multiple of $G$ with the least positive leading coefficient. Thus we have (34) and infer, by (32) and (18), that $K F=L F$.

Hence in any case Theorem 1 applies to $F$. By virtue of that theorem and of (34) there exist a matrix $\boldsymbol{M} \in \mathfrak{M}_{k, k}(\mathbb{Z})$ and a vector $\boldsymbol{v} \in \mathbb{Z}^{k}$ such that (26), with $c_{11}(G)=9 k_{0} \cdot 2^{\|F\|-5}$, and (27) hold and either

$$
K N_{\boldsymbol{K} / \mathbb{Q}} G\left(\boldsymbol{z}^{\boldsymbol{M}}\right) \stackrel{\text { can }}{=} \text { const } \prod_{\sigma=1}^{s} F_{\sigma}(\boldsymbol{z})^{e_{\sigma}}
$$

implies

$$
K N_{K / \mathbb{Q}} G\left(x^{n}\right) \stackrel{\text { can }}{=} \text { const } \prod_{\sigma=1}^{s} F_{\sigma}\left(x^{v}\right)^{e_{\sigma}}
$$

or there exists a $\gamma_{1} \in \mathbb{Z}^{k}$ such that

$$
\boldsymbol{\gamma}_{1} \boldsymbol{n}=0 \quad \text { and } \quad 0<h\left(\boldsymbol{\gamma}_{1}\right) \leq c_{3}(F, \boldsymbol{M})=c_{13}(G, \boldsymbol{M}) \text {. }
$$

In the latter case we have (29) provided

$$
c_{12}(G) \geq \max c_{13}(G, M),
$$

where the maximum is taken over all matrices $\boldsymbol{M} \in \mathfrak{M}_{k, k}(\mathbb{Z})$ satisfying (26). In the former case on the right-hand side of (36) we have $\sum_{\sigma=1}^{s} e_{\sigma} \geq 1$. Indeed, if $\boldsymbol{K} \neq \mathbb{Q}$, then by Lemma 3,

$$
L F\left(\boldsymbol{z}^{\boldsymbol{M}}\right)=J F\left(\boldsymbol{z}^{\boldsymbol{M}}\right),
$$


hence by (18),

$$
K F\left(\boldsymbol{z}^{M}\right)=J F\left(\boldsymbol{z}^{M}\right) \notin \mathbb{Q} .
$$

If $\boldsymbol{K}=\mathbb{Q}$ the same argument works with $F$ replaced by $K G$.

If $\sum_{\sigma=1}^{s} e_{\sigma}=1$, then by (37), $K N_{\boldsymbol{K} / \mathbb{Q}} G\left(x^{n}\right)$ is irreducible, hence we have (28). If $\sum_{\sigma=1}^{s} e_{\sigma} \geq 2$, then we have (30). Indeed, otherwise $J G\left(\boldsymbol{z}^{M}\right)$ would be irreducible over $\widehat{\boldsymbol{K}}$ and would satisfy

$$
J G\left(\boldsymbol{z}^{\boldsymbol{M}}\right) \mid F_{\sigma}(\boldsymbol{z})
$$

for a $\sigma \leq s$. Since

$$
J G\left(x^{n}\right)=J G\left(\left(x^{v}\right)^{\boldsymbol{M}}\right),
$$

(34) implies that $J G\left(\boldsymbol{z}^{\boldsymbol{M}}\right)^{\tau_{2}} / J G\left(\boldsymbol{z}^{\boldsymbol{M}}\right)^{\tau_{1}} \notin \widehat{\boldsymbol{K}}$ for any two distinct elements $\tau_{1}, \tau_{2}$ of $T$. Since $J G\left(\boldsymbol{z}^{\boldsymbol{M}}\right)^{\tau_{1}}, J G\left(\boldsymbol{z}^{\boldsymbol{M}}\right)^{\tau_{2}}$ are both irreducible over $\widehat{\boldsymbol{K}}$,

$$
\left(J G\left(\boldsymbol{z}^{M}\right)^{\tau_{1}}, J G\left(\boldsymbol{z}^{M}\right)^{\tau_{2}}\right)=1
$$

and by (38),

$$
J N_{\boldsymbol{K} / \mathbb{Q}} G\left(\boldsymbol{z}^{\boldsymbol{M}}\right)=\prod_{\tau \in T} J G\left(\boldsymbol{z}^{\boldsymbol{M}}\right)^{\tau} \mid F_{\sigma}(\boldsymbol{z}),
$$

contrary to (36) under the assumption $\sum_{\sigma=1}^{s} e_{\sigma} \geq 2$. The contradiction obtained shows (30). If $\boldsymbol{K}=\mathbb{Q}$ the same assumption together with (37) shows the existence of a factorization (30) satisfying (31). Indeed, according to the definition of canonical factorization, $F_{\sigma}\left(x^{v}\right) \notin \mathbb{Q}$ for all $\sigma \leq s$.

Proof of Theorem 2. The reducibility condition given in the theorem is clearly sufficient. We proceed to prove that it is necessary. Assume that the condition is necessary for $\mathbb{Q}\left[x_{1}, \ldots, x_{k-1}\right], c_{4}(F)$ being defined for all polynomials in less than $k$ variables for which it is needed (for $k=1$ this is an empty statement); assume that $F \in \mathbb{Q}[\boldsymbol{x}], J F\left(x^{n}\right)$ is not reciprocal and $K F\left(x^{n}\right)$ is reducible.

Consider first the case where $F$ is of positive degree with respect to all $k$ variables, so that $k$ is determined by $F$. For $k=1$ this is the only case.

If the matrix $\boldsymbol{M}$ and the vector $\boldsymbol{v}$ appearing in Lemma 8 for $G=F$ have the properties (30) and (31) we take $\boldsymbol{N}=\boldsymbol{M}, r=k, F_{i}=\left(K F, G_{i}\right)$ $(i=1,2)$ and obtain $h(\boldsymbol{N}) \leq c_{11}(F)$. Otherwise, by Lemma 8, there exists a vector $\boldsymbol{\gamma} \in \mathbb{Z}^{k}$ such that $\boldsymbol{\gamma} \boldsymbol{n}=0$ and $0<h(\gamma) \leq c_{12}(F)$. For $k=1$ this completes the proof, since $\boldsymbol{\gamma} \boldsymbol{n}=0$ implies $\boldsymbol{n}=\mathbf{0}$.

For $k>1$ the integer vectors perpendicular to $\gamma$ form a lattice, say $\boldsymbol{\Lambda}$. It is easily seen (cf. for instance Lemma 6 in [2]) that $\boldsymbol{\Lambda}$ has a basis that written in the form of a matrix $\boldsymbol{B} \in \mathfrak{M}_{k-1, k}(\mathbb{Z})$ satisfies

$$
h(\boldsymbol{B}) \leq \frac{k}{2} c_{12}(F) .
$$


Let us put

$$
\widetilde{F}=J F\left(\widetilde{\boldsymbol{x}}^{\boldsymbol{B}}\right), \quad \text { where } \widetilde{\boldsymbol{x}}=\left[x_{1}, \ldots, x_{k-1}\right] .
$$

Since $\boldsymbol{n} \in \boldsymbol{\Lambda}$ we have $\boldsymbol{n}=\boldsymbol{m} \boldsymbol{B}$ for an $\boldsymbol{m} \in \mathbb{Z}^{k-1}$. Clearly

$$
J F\left(x^{n}\right)=J \widetilde{F}\left(x^{m}\right),
$$

thus, by assumption, $J \widetilde{F}\left(x^{m}\right)$ is not reciprocal and $K \widetilde{F}\left(x^{m}\right)$ is reducible. By the inductive assumption there exist a matrix $\widetilde{N} \in \mathfrak{M}_{r, k-1}(\mathbb{Z})$ of rank $r \leq k-1$ and a vector $\boldsymbol{v} \in \mathbb{Z}^{r}$ such that

$$
\begin{gathered}
h(\widetilde{\boldsymbol{N}}) \leq c_{4}(\widetilde{F}), \\
\boldsymbol{m}=\boldsymbol{v} \widetilde{\boldsymbol{N}} ; \\
K \widetilde{F}\left(\boldsymbol{y}^{\widetilde{N}}\right)=F_{1} F_{2}, \quad F_{i} \in \mathbb{Q}[\boldsymbol{y}], \quad K F_{i}\left(x^{\boldsymbol{v}}\right) \notin \mathbb{Q} \quad(i=1,2) .
\end{gathered}
$$

Let us take $\boldsymbol{N}=\widetilde{\boldsymbol{N}} \boldsymbol{B}$. It follows from (40) that $J \widetilde{F}\left(\boldsymbol{y}^{\widetilde{N}}\right)=J F\left(\boldsymbol{y}^{\boldsymbol{N}}\right)$ and from (43) that $\boldsymbol{n}=\boldsymbol{v} \boldsymbol{N}$; moreover, since rank $\boldsymbol{B}=k-1$, rank $\boldsymbol{N}=r$. Thus $\boldsymbol{N}$ and $\boldsymbol{v}$ have all the properties required in the theorem apart from (6); it remains to establish (6) by an appropriate choice of $c_{4}(F)$. We have, by (39) and (42),

$$
h(\boldsymbol{N}) \leq(k-1) h(\widetilde{\boldsymbol{N}}) h(\boldsymbol{B}) \leq\left(\begin{array}{l}
k \\
2
\end{array}\right) c_{4}(\widetilde{F}) c_{12}(F) .
$$

However, $\widetilde{F}$ is determined by $F$ and $\boldsymbol{B}$ via (40) and, by virtue of (39), $\boldsymbol{B}$ runs through a finite set of matrices depending only on $F$. Hence $c_{4}(\widetilde{F}) \leq c_{14}(F)$ and the theorem holds with

$$
c_{4}(F)=\max \left\{c_{11}(F),\left(\begin{array}{l}
k \\
2
\end{array}\right) c_{12}(F) c_{14}(F)\right\} .
$$

Consider now the case where $F$ is of positive degree with respect to less than $k$ variables. We may assume that $F \in \mathbb{Q}[\widetilde{\boldsymbol{x}}]$. By the inductive assumption there exist a matrix $\boldsymbol{N}_{0} \in \mathfrak{M}_{k-1, r_{0}}(\mathbb{Z})$ of rank $r_{0}$ and a vector $\boldsymbol{v}_{0} \in \mathbb{Z}^{r_{0}}$ such that

$$
\begin{gathered}
h\left(\boldsymbol{N}_{0}\right) \leq c_{4}(F), \quad\left[n_{1}, \ldots, n_{k}\right]=\boldsymbol{v}_{0} \boldsymbol{N}_{0}, \\
K F\left(\boldsymbol{y}_{0}^{\boldsymbol{N}_{0}}\right)=F_{1} F_{2}, \quad \boldsymbol{y}_{0}=\left[y_{1}, \ldots, y_{r_{0}}\right], \\
F_{i} \in \mathbb{Q}\left[\boldsymbol{y}_{0}\right], \quad K F_{i}\left(x^{\boldsymbol{v}_{0}}\right) \notin \mathbb{Q} \quad(i=1,2) .
\end{gathered}
$$

We put $r=r_{0}+1, \boldsymbol{N}=\left(\begin{array}{cc}\boldsymbol{N}_{0} & 0 \\ 0 & 1\end{array}\right), \boldsymbol{v}=\left[\boldsymbol{v}_{0}, n_{k}\right]$ and easily verify that conditions (6)-(9) are satisfied.

Proof of Theorem 3. We proceed in the same way as in the proof of the necessity part of Theorem 2 , with $\boldsymbol{K}$ instead of $\mathbb{Q}$, using Lemma 8 without the formula (31). Therefore we point out only the argument not needed in the proof of Theorem 2. Before applying the inductive assumption to $\widetilde{F}\left(x^{m}\right)$ 
we have to check that $\widetilde{F} \in \boldsymbol{K}[\widetilde{\boldsymbol{x}}]$ and that

$$
\frac{J \widetilde{F}\left(\widetilde{\boldsymbol{x}}^{-\boldsymbol{m}}\right)}{J \widetilde{F}^{\tau}\left(\widetilde{\boldsymbol{x}}^{m}\right)} \notin \widehat{\boldsymbol{K}}
$$

for all embeddings $\tau$ of $\boldsymbol{K}$ into $\widehat{\boldsymbol{K}}$.

Now $\widetilde{F} \in \boldsymbol{K}[\widetilde{\boldsymbol{x}}]$ follows from $F \in \boldsymbol{K}[\boldsymbol{x}]$ and from the definition of $\widetilde{F}$ by the formula (40), while (44) follows from (10) and (41).

Lemma 9. If $a_{j} \neq 0(0 \leq j \leq k)$ are complex numbers and the rank of a matrix $\left(\nu_{i j}\right) \in \mathfrak{M}_{r, k}(\mathbb{Z})$ is greater than $(k+1) / 2$, then

$$
J\left(a_{0}+\sum_{j=1}^{k} a_{j} \prod_{i=1}^{r} x_{i}^{\nu_{i j}}\right)
$$

is absolutely irreducible.

Proof. See [3], Corollary to Theorem 1. The proof of Theorem 1 given there shows less than stated in the theorem, but only in the case of positive characteristic of the ground field, so the Corollary is fully justified.

Proof of Corollary 1. We apply Theorem 3 with $F=a_{0}+\sum_{j=1}^{k} a_{j} x_{j}$ and infer that if $K\left(a_{0}+\sum_{j=1}^{k} a_{j} x^{n_{j}}\right)$ is irreducible over $\boldsymbol{K}$, then either

$$
\frac{J\left(a_{0}+\sum_{j=1}^{k} a_{j} x^{-n_{j}}\right)}{a_{0}^{\tau}+\sum_{j=1}^{k} a_{j}^{\tau} x^{n_{j}}} \in \widehat{\boldsymbol{K}}
$$

for an embedding $\tau$ of $\boldsymbol{K}$ into $\widehat{\boldsymbol{K}}$, or there exist a matrix $\boldsymbol{N}=\left(\nu_{i j}\right) \in$ $\mathfrak{M}_{r, k}(\mathbb{Z})$ of rank $r$ and a vector $\boldsymbol{v} \in \mathbb{Z}^{r}$ such that $h(\boldsymbol{N}) \leq c_{4}(F), \boldsymbol{n}=\boldsymbol{v} \boldsymbol{N}$ and

$$
J\left(a_{0}+\sum_{j=1}^{k} a_{j} \prod_{i=1}^{r} y_{i}^{\nu_{i j}}\right) \text { is reducible over } \widehat{\boldsymbol{K}} .
$$

Let us put $c_{6}(\boldsymbol{a})=\max \left\{2, c_{4}(F)\right\}$.

If (45) holds, then $n_{j}+n_{k-j}=n_{k}(1 \leq j<k)$ and we satisfy (13) and (14) by taking

$$
\begin{aligned}
& \boldsymbol{v}_{0}= \begin{cases}{\left[n_{1}, \ldots, n_{k / 2}\right]} & \text { if } k \text { is even, } \\
{\left[n_{1}, \ldots, n_{(k-1) / 2}, n_{k}\right]} & \text { if } k \text { is odd }\end{cases} \\
& \boldsymbol{N}_{0}=\left(\begin{array}{cccccccccc}
1 & & & & & & & & -1 & \\
& 1 & & & & & & . \cdot & & \\
& & \ddots & & & & & & & \\
& & & 1 & & -1 & & & & \\
& & & & 1 & 2 & 2 & \ldots & 2 & 2
\end{array}\right) \quad \text { if } k \text { is even, }
\end{aligned}
$$




$$
\boldsymbol{N}_{0}=\left(\begin{array}{ccccccccc}
1 & & & & & & & & -1 \\
& 1 & & & & & . & & \\
& & \ddots & & & -1 & & & \\
& & & 1 & 1 & & & & \\
& & & & 1 & 1 & \ldots & 1 & 1
\end{array}\right) \quad \text { if } k \text { is odd }
$$

where the empty places (but not the dots) denote zeros.

If (46) holds, then by Lemma $9, r \leq(k+1) / 2$. If $r=[(k+1) / 2]$ we take $\boldsymbol{N}_{0}=\boldsymbol{N}, \boldsymbol{v}_{0}=\boldsymbol{v}$; if $r<(k+1) / 2$ we amplify $\boldsymbol{N}$ and $\boldsymbol{v}$ by inserting zeros.

Proof of Corollary 2. For each matrix $\boldsymbol{N}_{0} \in \mathfrak{M}_{[(k+1) / 2], k}(\mathbb{Z})$ the number of vectors $\boldsymbol{n} \in \mathbb{Z}^{k}$ with $h(\boldsymbol{n}) \leq N$ for which there exists a $\boldsymbol{v}_{0} \in \mathbb{Z}^{[(k+1) / 2]}$ satisfying (14) is less than $c_{15}\left(\overline{\boldsymbol{N}}_{0}\right) N^{[(k+1) / 2]}$. Hence Corollary 2 follows from Corollary 1 with

$$
c_{7}(\boldsymbol{a})=\sum c_{15}\left(\boldsymbol{N}_{0}\right)
$$

where the sum is taken over all matrices $\boldsymbol{N}_{0} \in \mathfrak{M}_{[(k+1) / 2], k}$ satisfying (13).

REMARK 1. If $k>1$ and $\sum_{j=0}^{k} a_{j}=0$, then the polynomial $a_{0}+$ $\sum_{j=1}^{k} a_{j} x^{n_{j}}$ is reducible for all vectors $\boldsymbol{n}$ in question. This shows that replacing $a_{0}+\sum_{j=1}^{k} a_{j} x^{n_{j}}$ by $K\left(a_{0}+\sum_{j=1}^{k} a_{j} x^{n_{j}}\right)$ is really needed in order to obtain a non-trivial result.

EXAMPLE. Here is the example announced in the introduction showing that the exponent $[(k+1) / 2]$ is best possible in Corollary 2 , and hence also in Corollary 1.

If $k=2 l-1$ we take $a_{0}=4, a_{j}=2(1 \leq j \leq l), a_{j}=1(l<j<2 l)$, $n_{j}=n_{l}+n_{j-l}(l<j<2 l)$. It follows that

$$
a_{0}+\sum_{j=1}^{k} a_{j} x^{n_{j}}=\left(2+\sum_{j=1}^{l-1} x^{n_{j}}\right)\left(2+x^{n_{l}}\right) .
$$

The two factors on the right-hand side are not reciprocal, hence $K\left(a_{0}+\right.$ $\left.\sum_{j=1}^{k} a_{j} x^{n_{j}}\right)$ is reducible. The number $X$ of relevant vectors $\boldsymbol{n}$ with $n_{k} \leq N$ is at least equal to the number of increasing sequences $n_{1}<\ldots<n_{l}$ with $n_{l} \leq[N / 2]$, hence

$$
X \geq\left(\begin{array}{c}
{[N / 2]} \\
l
\end{array}\right) \geq c_{16}(l) N^{l} \quad \text { for } N \geq 2 l,
$$

where $c_{16}(l)>0$.

If $k=2 l$ we take $a_{0}=4, a_{j}=2(1 \leq j \leq l), a_{l+1}=3, a_{j}=1$ $(l+1<j \leq 2 l), n_{j}=n_{l}+n_{j-l}(l<j<2 l), n_{2 l}=2 n_{l}+n_{1}$. It follows that

$$
a_{0}+\sum_{j=1}^{k} a_{j} x^{n_{j}}=\left(2+\sum_{j=1}^{l-1} x^{n_{j}}+x^{n_{l}+n_{1}}\right)\left(2+x^{n_{l}}\right) .
$$


The two factors on the right-hand side are not reciprocal, hence $K\left(a_{0}+\right.$ $\left.\sum_{j=1}^{k} a_{j} x^{n_{j}}\right)$ is reducible. The number $X$ of relevant vectors $\boldsymbol{n}$ with $n_{k} \leq N$ is at least equal to the number of increasing sequences $n_{1}<\ldots<n_{l}$ with $n_{l} \leq[N / 3]$, hence

$$
X \geq\left(\begin{array}{c}
{[N / 3]} \\
l
\end{array}\right) \geq c_{17}(l) N^{l} \quad \text { for } N \geq 3 l,
$$

where $c_{17}(l)>0$.

Lemma 10. For any $k+1$ non-zero complex numbers $a_{0}, \ldots, a_{k}$ such that $a_{0} \in \boldsymbol{K}=\mathbb{Q}\left(a_{1} / a_{0}, \ldots, a_{k} / a_{0}\right)$ there exist $k+1$ algebraic numbers $\alpha_{0}, \ldots, \alpha_{k-1}, \alpha_{k}=1$ such that if $0=n_{0}<n_{1}<\ldots<n_{k}$ and $K\left(\sum_{j=0}^{l} a_{j} x^{n_{j}}\right)$ is reducible over $\boldsymbol{K}$ then either $K\left(\sum_{j=0}^{l} \alpha_{j} x^{n_{j}}\right)$ is reducible over $\boldsymbol{K}_{0}=\mathbb{Q}\left(\alpha_{0}, \ldots, \alpha_{k-1}\right)$, or there is a vector $\boldsymbol{\gamma} \in \mathbb{Z}^{k}$ such that $\boldsymbol{\gamma} \boldsymbol{n}=0$ and

$$
0<h(\gamma) \leq c_{18}(\boldsymbol{a})
$$

Proof. See [6], Lemma 5.

Proof of Corollary 3. Let $\alpha_{i}$ have the meaning of Lemma 10. By Corollary 2 the number of relevant vectors $\boldsymbol{n}$ for which $n_{k} \leq N$ and $K\left(\sum_{j=0}^{k} \alpha_{j} x^{n_{j}}\right)$ is reducible over $\mathbb{Q}\left(\alpha_{0}, \ldots, \alpha_{k-1}\right)$ is less than $c_{7}(\boldsymbol{\alpha}) N^{[(k+1) / 2]}$. For a fixed $\boldsymbol{\alpha} \in \mathbb{Z}^{k} \backslash\{\mathbf{0}\}$ the number of relevant vectors $\boldsymbol{n} \in \mathbb{Z}^{k}$ with $n_{k} \leq N$ such that $\boldsymbol{\gamma} \boldsymbol{n}=0$ is less than $c_{19}(\boldsymbol{\gamma}) N^{k-1}$. Hence Corollary 3 holds with

$$
c_{8}(\boldsymbol{a})=c_{7}(\boldsymbol{\alpha})+\sum c_{19}(\boldsymbol{\gamma})
$$

where the sum is taken over all vectors $\gamma \in \mathbb{Z}^{k}$ satisfying (47).

REMARK 2. It seems likely that by improving Lemma 10 one can replace the exponent $k-1$ in Corollary 3 by $[(k+1) / 2]$.

Proof of Theorem 4. We begin by defining subsets $S_{i}$ and $R_{i}$ of $\mathfrak{M}_{k-i, k}(\mathbb{Z})$ $(0 \leq i<k)$ inductively, as follows:

$$
S_{0}=\left\{\boldsymbol{I}_{k}\right\},
$$

and supposing that $S_{i}$ is already defined and $\boldsymbol{y}=\left[y_{1}, \ldots, y_{k-i}\right]$,

$$
\begin{array}{r}
R_{i}=\left\{\boldsymbol{M N}: \boldsymbol{N} \in S_{i}, \boldsymbol{M} \in \mathfrak{M}_{k-i, k-i}(\mathbb{Z}), \operatorname{det} \boldsymbol{M} \neq 0,\right. \\
\left.h(\boldsymbol{M}) \leq c_{11}\left(F\left(\boldsymbol{y}^{\boldsymbol{N}}\right)\right), K F\left(\boldsymbol{y}^{\boldsymbol{M}}\right) \text { is reducible }\right\},
\end{array}
$$

and for $i<k-1$,

$$
\begin{aligned}
S_{i+1}=\left\{\boldsymbol{N} \in \mathfrak{M}_{k-i-1, k}(\mathbb{Z}): \operatorname{rank} \boldsymbol{N}=k-i-1,\right. \\
h(\boldsymbol{N}) \leq \frac{1}{2}(k-i)^{2} \max _{\boldsymbol{N}_{1} \in S_{i}}\left\{h ( \boldsymbol { N } _ { 1 } ) \operatorname { m a x } \left\{\max c_{12}\left(F\left(\boldsymbol{y}^{\boldsymbol{N}_{1}}\right)\right),\right.\right. \\
\left.\left.\left.\max ^{*}(k-1) c_{10}(D) h(\boldsymbol{M})\right\}\right\}\right\}
\end{aligned}
$$


where $\max ^{*}$ is taken over all $\boldsymbol{M} \in \mathfrak{M}_{k-i, k-i}(\mathbb{Z})$ with $\operatorname{det} \boldsymbol{M} \neq 0, h(\boldsymbol{M}) \leq$ $c_{11}\left(F\left(\boldsymbol{y}^{N_{1}}\right)\right.$ ) and all monic irreducible divisors $D$ of $K F\left(\boldsymbol{y}^{M N_{1}}\right)$. (If $K F\left(\boldsymbol{y}^{M N_{1}}\right) \in \mathbb{Q}$ we take $\max ^{*}=0$.)

In this way $R_{i}$ and $S_{i}$ are defined for all $i<k$ and we put

$$
R=\bigcup_{i=0}^{k-1} R_{i}, \quad S=\bigcup_{i=1}^{k-1} S_{i} .
$$

We first prove that the condition given in the theorem is necessary. By (48) there exist indices $i$ such that

$$
\boldsymbol{n}=\boldsymbol{u} \boldsymbol{U}, \quad \boldsymbol{U} \in S_{k-i}, \quad \boldsymbol{u} \in \mathbb{Z}^{i} .
$$

Let $r$ be the least such index and

$$
\boldsymbol{n}=\boldsymbol{v} \boldsymbol{N}, \quad \boldsymbol{N} \in S_{k-r}, \quad \boldsymbol{v} \in \mathbb{Z}^{r} .
$$

By Lemma 8 if $K F\left(x^{n}\right)=K F\left(x^{v N}\right)$ is reducible, then there exists a matrix $\boldsymbol{M} \in \mathfrak{M}_{r, r}(\mathbb{Z})$ such that

$$
\begin{gathered}
\operatorname{det} \boldsymbol{M} \neq 0, \quad h(\boldsymbol{M}) \leq c_{11}\left(F\left(\boldsymbol{y}^{\boldsymbol{N}}\right)\right), \quad \boldsymbol{y}=\left[y_{1}, \ldots, y_{r}\right], \\
\boldsymbol{v}=\boldsymbol{v}_{1} \boldsymbol{M}, \quad \boldsymbol{v}_{1} \in \mathbb{Z}^{r}
\end{gathered}
$$

and either $K F\left(\boldsymbol{y}^{M N}\right)$ is reducible, or there exists a vector $\gamma \in \mathbb{Z}^{r}$ such that

$$
\boldsymbol{\gamma} \boldsymbol{v}=0 \quad \text { and } \quad 0<h(\boldsymbol{\gamma}) \leq c_{12}\left(F\left(\boldsymbol{y}^{\boldsymbol{N}}\right)\right) \text {. }
$$

The second possibility can only hold for $r>1$ since for $r=1$ it gives $\boldsymbol{v}=\mathbf{0}$ and by (51), $\boldsymbol{n}=\mathbf{0}$. For $r>1$ the vectors $\boldsymbol{v}$ perpendicular to $\boldsymbol{\gamma}$ form a lattice $\boldsymbol{\Lambda}$ in $\mathbb{Z}^{r}$. This lattice has a basis that written in the form of a matrix $\boldsymbol{B} \in \mathfrak{M}_{r-1, r}(\mathbb{Z})$ satisfies

$$
\begin{aligned}
\operatorname{rank} \boldsymbol{B} & =r-1, \\
h(\boldsymbol{B}) \leq \frac{r}{2} h(\boldsymbol{\gamma}) & \leq \frac{r}{2} c_{12}\left(F\left(\boldsymbol{y}^{\boldsymbol{N}}\right)\right)
\end{aligned}
$$

(cf. Lemma 6 in [2]). Since $\boldsymbol{v} \in \boldsymbol{\Lambda}$ we have

$$
\boldsymbol{v}=\boldsymbol{w} \boldsymbol{B}, \quad \boldsymbol{w} \in \mathbb{Z}^{r-1}
$$

hence, by (51),

$$
\boldsymbol{n}=\boldsymbol{w} \boldsymbol{B} \boldsymbol{N}, \quad \boldsymbol{B} \boldsymbol{N} \in \mathfrak{M}_{r-1, k}(\mathbb{Z}) .
$$

Since, by (50) and (51), rank $\boldsymbol{N}=r$, it follows from (54), by linear algebra, that

$$
\operatorname{rank} \boldsymbol{B} \boldsymbol{N}=r-1 .
$$

Moreover, by (55),

$$
h(\boldsymbol{B N}) \leq r h(\boldsymbol{B}) h(\boldsymbol{N}) \leq \frac{r^{2}}{2} h(\boldsymbol{N}) c_{12}\left(F\left(\boldsymbol{y}^{\boldsymbol{N}}\right)\right)
$$

and, by (50), $\boldsymbol{B} \boldsymbol{N} \in S_{k-r+1}$, contrary, in view of (56), to the definition 
of $r$. The contradiction obtained proves that $K F\left(\boldsymbol{y}^{M N}\right)$ is reducible, hence $\boldsymbol{M N} \in R_{k-r}$ by (49). By (51) and (53) we have

$$
n=v_{1} M N,
$$

while by the definition of $r$ the equation $\boldsymbol{n}=\boldsymbol{u} \boldsymbol{U}$ in unsoluble in $\boldsymbol{u} \in \mathbb{Z}^{i}$, $\boldsymbol{U} \in S_{k-i}$ for $i<r$. Thus the condition given in the theorem is necessary.

Now we prove that it is sufficient. Assume that for a certain matrix $N \in R_{k-r}(1 \leq r \leq k)$,

$$
\boldsymbol{n}=\boldsymbol{v} \boldsymbol{N}, \quad \boldsymbol{v} \in \mathbb{Z}^{r}
$$

but

$$
\boldsymbol{n} \neq \boldsymbol{u} \boldsymbol{U} \quad \text { for all } s<r, \quad \boldsymbol{u} \in \mathbb{Z}^{s}, \quad \boldsymbol{U} \in S_{k-s} .
$$

Then by (49),

$$
\begin{gathered}
\boldsymbol{n}=\boldsymbol{v} \boldsymbol{M} \boldsymbol{N}_{1}, \quad \boldsymbol{N}_{1} \in S_{k-r}, \quad \boldsymbol{M} \in \mathfrak{M}_{r, r}(\mathbb{Z}), \quad \operatorname{det} \boldsymbol{M} \neq 0, \\
h(\boldsymbol{M}) \leq c_{11}\left(F\left(\boldsymbol{y}^{\boldsymbol{N}_{1}}\right)\right), \quad \boldsymbol{y}=\left[y_{1}, \ldots, y_{r}\right]
\end{gathered}
$$

and

Hence

$$
K F\left(\boldsymbol{y}^{M N_{1}}\right)=F_{1} F_{2}, \quad F_{1}, F_{2} \in \mathbb{Q}[\boldsymbol{y}] \backslash \mathbb{Q} .
$$

$$
K F\left(x^{n}\right)=K F_{1}\left(x^{v}\right) K F_{2}\left(x^{v}\right) .
$$

Suppose that for an $i \leq 2$ we have $K F_{i}\left(x^{\boldsymbol{v}}\right) \in \mathbb{Q}$. Then $K D\left(x^{\boldsymbol{v}}\right) \in \mathbb{Q}$ for an irreducible monic factor $D$ of $K F$, hence by Lemma 7 there exists a vector $\gamma \in \mathbb{Z}^{r}$ such that

$$
\boldsymbol{\gamma} \boldsymbol{v}=0, \quad 0<h(\boldsymbol{\gamma}) \leq c_{10}(D) .
$$

Again this can occur only for $r>1$ and, repeating the argument about the lattice given above, we find a matrix $\boldsymbol{B} \in \mathfrak{M}_{r-1, r}(\mathbb{Z})$ such that

$$
\begin{gathered}
\operatorname{rank} \boldsymbol{B}=r-1, \quad h(\boldsymbol{B}) \leq \frac{r}{2} h(\boldsymbol{\gamma}) \leq \frac{r}{2} c_{10}(D) ; \\
\boldsymbol{v}=\boldsymbol{w} \boldsymbol{B}, \quad \boldsymbol{w} \in \mathbb{Z}^{r-1} .
\end{gathered}
$$

It follows that

$$
\begin{gathered}
\boldsymbol{n}=\boldsymbol{w} \boldsymbol{B} \boldsymbol{M} \boldsymbol{N}_{1}, \quad \boldsymbol{B} \boldsymbol{M} \boldsymbol{N}_{1} \in \mathfrak{M}_{r-1, k}(\mathbb{Z}), \\
\operatorname{rank} \boldsymbol{B} \boldsymbol{M} \boldsymbol{N}_{1}=r-1, \\
h\left(\boldsymbol{B} \boldsymbol{M} \boldsymbol{N}_{1}\right) \leq r^{2} h(\boldsymbol{B}) h(\boldsymbol{M}) h\left(\boldsymbol{N}_{1}\right) \leq \frac{r^{3}}{2} c_{10}(D) h(\boldsymbol{M}) h\left(\boldsymbol{N}_{1}\right),
\end{gathered}
$$

hence by (50),

$$
\boldsymbol{B} \boldsymbol{M} \boldsymbol{N}_{1} \in S_{k-r+1},
$$

which together with (59) contradicts (58). The contradiction obtained shows that $K F_{i}\left(x^{v}\right) \notin \mathbb{Q}(i=1,2)$, hence by $(59), K F\left(x^{n}\right)$ is reducible. 


\section{References}

[1] E. Bombieri and U. Zannier, Intersections of varieties with 1-dimensional tori and a conjecture of Schinzel, preprint; see also U. Zannier, Proof of Conjecture 1, appendix in the book by A. Schinzel, Polynomials with Special Regard to Reducibility, to be published by Cambridge University Press.

[2] A. Schinzel, Reducibility of lacunary polynomials I, Acta Arith. 16 (1969), 123-159.

[3] -, A general irreducibility criterion, J. Indian Math. Soc. (N.S.) 37 (1973), 1-8.

[4] -, Reducibility of lacunary polynomials III, Acta Arith. 34 (1978), 227-266.

[5] - Reducibility of lacunary polynomials VII, Monatsh. Math. 102 (1986), 309-337.

[6] -, Reducibility of lacunary polynomials VIII, Acta Arith. 50 (1988), 91-106.

[7] - Reducibility of lacunary polynomials X, ibid. 53 (1989), 47-97.

[8] —, Reducibility of lacunary polynomials XI, ibid. 57 (1991), 165-175.

Institute of Mathematics

Polish Academy of Sciences

P.O. Box 137

00-950 Warszawa, Poland

E-mail: schinzel@impan.gov.pl 